\title{
INVESTIGACIÓN/RESEARCH
}

Recibido: 28/05/2014---Revisado: 22/06/2014

Aceptado: 01/07/2014---Publicado: 15/07/2014

\section{ELEMENTOS ASOCIADOS AL IDIOMA MAPUZUNGUN QUE FACILITAN Y/U OBSTACULIZAN EL PROCESO DE APRENDIZAJE DEL IDIOMA INGLÉS EN ESTUDIANTES DE ORIGEN MAPUCHE}

Juan Alfredo del Valle Rojas. Universidad Católica de Temuco. Chile. dvalle1985@hotmail.com

César Eduardo Soto Vallejos. Universidad Católica de Temuco. Chile. csoto@hotmail.com

José Luis Sandoval Tripailaf. Universidad Católica de Temuco. Chile.

elpashe@hotmail.com

Joaquín Eduardo Otárola Rodríguez. Universidad Católica de Temuco. Chile. juaco 1984@hotmail.com

Misael Andrés Valdés Guzmán. Universidad Católica de Temuco. Chile. misaelicious@live.com

Harold Hernán Sanhueza Rodríguez. Universidad Católica de Temuco. Chile. ecklesiastes@hotmail.com

\section{RESUMEN}

El objetivo de esta investigación es determinar cuáles son los elementos asociados al idioma mapuzungun que facilitan u obstaculizan el aprendizaje del idioma inglés. En este sentido, son diversos los factores que influyen en el aprendizaje del inglés como un tercer idioma. En relación a los elementos ligados a la cultura mapuche, se encontraron elementos que pudieron haber facilitado y obstaculizado el aprendizaje del inglés.

Los hallazgos permiten establecer que el elemento asociado al mapuzungun que facilitó el aprendizaje del inglés en los participantes es la Transferencia Lingüística, ya que este factor se identificó en cuatro de los seis casos. Ellos señalaron que al haber elementos parecidos entre los dos idiomas, aquello facilitó el proceso de aprendizaje del inglés. Igualmente, hubo otros factores que influyeron positivamente en el proceso de aprendizaje del inglés, tales como la Conciencia Metalingüística y factores socioculturales, los cuales se hallaron en tan sólo un caso. En cuanto a los factores asociados al mapuzungun que obstaculizaron el aprendizaje del inglés, se encontró que en la relación entre la cultura mapuche y occidental, existen elementos que obstaculizaron el proceso de aprendizaje en un participante. Por otra parte, se presentó el caso en el cual la diferencia de origen lingüístico del español, mapuzungun e inglés resultó ser un elemento obstaculizador en el aprendizaje del idioma inglés.

\section{PALABRAS CLAVE}


Bilingüismo - Trilingüismo - Lingua franca - Mapuche - Mapuzungun - Motivación

Intrínseca - Motivación Extrínseca - Conciencia Metalingüística - Transferencia

Lingüística

Bilingüismo - Trilingüismo - Lingua franca - Mapuche - Mapuzungun - Motivación Intrínseca

- Motivación Extrínseca - Conciencia Metalingüística - Transferencia Lingüística.

\title{
ELEMENTS ASSOCIATED TO MAPUZUNGUN LANGUAGE THAT FACILITATE AND/OR INTERFERE IN THE ENGLISH LANGUAGE LEARNING PROCESS IN MAPUCHE STUDENTS
}

\begin{abstract}
This research proposes the aim to determine the associated elements in mapuzungun language that facilitate and/or obstacle the learning process of english language. In this way, there are several factors that imply on the english language process as a third language. Regarding to the elements that are related to the mapuche culture, it was found out such elements that could have facilitated and obstructed english language learning process.

These findings state that the element associated to mapuzungun language that facilitates the english learning process in the participants (students interviewed in the research) is Language Transfer because this factor was found out in four out of six cases. The participants pointed out that the several elements similar in both languages (spanish and mapuzungun) facilitate the english learning process. Likely, there were some other factors that were found out in just one case that influenced positively the english learning process, such as: Metalinguistic Awareness and socio-cultural factors. Regarding to the factors associated to mapuzungun that obstructed the English learning process, there were found out the relationship between the mapuche and western culture. On the other hand, there was a finding that showed the difference in the linguistic origin of spanish, mapuzungun and english was an obstructed element in the english learning process.
\end{abstract}

\section{KEY WORDS}

Bilingualism - Trilingualism - Lingua franca - Mapuche - Mapuzungun - Intrinsic Motivation Extrinsic Motivation - Metalinguistic Awareness - Linguistic Transfer.

\section{INTRODUCCIÓN}

Durante los últimos años, la inserción de la Lengua Inglesa como idioma extranjero ha tomado gran importancia en el currículo de la educación chilena.

El Ministerio de Educación busca promover el idioma inglés promoviendo que todos los niños y niñas logren un dominio instrumental del idioma, para que así puedan hacer uso de él aumentando sus oportunidades tanto académicas como sus oportunidades de trabajo futuras. (MINEDUC, 1998)

La Región de la Araucanía no es ajena a la situación del país. Sin embargo, lo que caracteriza a esta Región es la gran cantidad de alumnos de origen mapuche que asisten a escuelas rurales cercanas a sus Comunidades o Reducciones en donde ellos viven, en las cuales en muchas ocasiones se utiliza el idioma propio de la etnia mapuche que es el 


$$
\begin{gathered}
\text { Bilingüismo - Trilingüismo - Lingua franca - Mapuche - Mapuzungun - Motivación } \\
\text { Intrínseca - Motivación Extrínseca - Conciencia Metalingüística - Transferencia } \\
\text { Lingüística }
\end{gathered}
$$

mapuzungun. Se puede señalar entonces que en la Región de la Araucanía existen algunas Instituciones de Educación Superior donde se imparte la carrera de Pedagogía en Inglés, la cual a su vez concentra una considerable cantidad de alumnos de origen mapuche.

Considerando el contexto en que se encuentran los estudiantes de Pedagogía en Inglés en la Universidad Católica de Temuco, y teniendo en cuenta el gran número de estudiantes mapuche en la Universidad, nos llevó a plantearnos acerca de cuáles, y cómo estos factores pudiesen afectar de manera positiva o negativa el aprendizaje del idioma inglés en aquellos alumnos.

\section{La enseñanza del idioma inglés en Chile}

La enseñanza del idioma inglés ha tomado gran auge durante las últimas décadas dentro de la educación chilena. En ese contexto, el 9 de junio de 2004 empezó a regir el Programa Inglés Abre Puertas del Ministerio de Educación. Este programa, lingüísticamente hablando, busca preparar a su población y convertir las dificultades en verdaderas oportunidades especialmente para alumnos y alumnas de generaciones futuras.

\section{La enseñanza del idioma inglés en la IX Región de la Araucanía}

El caso de la región de la Araucanía (IX Región de Chile), cuya realidad educacional manifiesta un alto grado de ruralidad, no ha constituido un obstáculo en cuanto a la enseñanza del idioma inglés. Por esa razón, un gran número de profesores pertenecientes a escuelas rurales han tomado la iniciativa de perfeccionar su nivel de inglés mediante el Programa Inglés Abre Puertas y de organizarse en una red de profesores rurales llamada Rural Teachers in the Net. (MINEDUC, 2009)

\section{La enseñanza del idioma inglés en la Universidad Católica de Temuco}

Considerando la importancia de la adquisición del idioma inglés, la Universidad Católica de Temuco muestra una particularidad en comparación a otras universidades del país, debido a que en esta región se da el caso de personas de origen mapuche que habitan un territorio que histórica y culturalmente se ha diferenciado al chileno.

La situación existente en la Novena Región, en el contexto de los estudiantes provenientes de zonas principalmente rurales que tienen nociones de su lengua ancestral y que además están aprendiendo inglés, coincide con el trabajo desarrollado en universidades del País Vasco, en el sentido que se intenta enseñar el inglés a estudiantes que poseen nociones de su lengua materna. De acuerdo a estudios llevados a cabo en el País Vasco, se puede señalar que la persona bilingüe cuenta con mayores ventajas, en comparación con un hablante monolingüe, al momento de enfrentarse al aprendizaje de un tercer idioma, debido a que el conocer previamente dos idiomas le ha permitido desarrollar estrategias para el aprendizaje de un tercero (Lasagabaster, 2005).

\section{OBJETIVOS}

Considerando el contexto en que se encuentran los estudiantes de Pedagogía en Inglés en la Universidad Católica de Temuco, y teniendo en cuenta el gran número de estudiantes 


$$
\begin{gathered}
\text { Bilingüismo - Trilingüismo - Lingua franca - Mapuche - Mapuzungun - Motivación } \\
\text { Intrínseca - Motivación Extrínseca - Conciencia Metalingüística - Transferencia } \\
\text { Lingüística }
\end{gathered}
$$

mapuche en la Universidad, nos llevó a plantearnos acerca de cuáles, y cómo estos factores pudiesen afectar de manera positiva o negativamente en el aprendizaje del idioma inglés en aquellos alumnos. En base a esto, la pregunta que surgió, y a la vez guió esta investigación es:

¿Cómo los elementos asociados al idioma mapuzungun presentes en estudiantes de la Universidad Católica de Temuco que tienen origen mapuche y además poseen nociones de mapuzungun pudiesen facilitar y/u obstaculizar el proceso de aprendizaje del idioma inglés?

- Se entiende por búsqueda de elementos facilitadores la comprensión de aquellos factores que conducen a los participantes a aprender el idioma inglés.

- $\quad$ Se entiende por la búsqueda de elementos obstaculizadores la comprensión de los factores que influyen de manera negativa en el aprendizaje del idioma inglés.

De lo expuesto anteriormente, se desprendió el siguiente objetivo general:

Objetivo general:

$>$ Establecer y comprender elementos asociados al idioma mapuzungun presentes en estudiantes de origen mapuche de la Universidad Católica de Temuco que poseen nociones de mapuzungun, los cuales pudiesen facilitar y/u obstaculizar su proceso de aprendizaje del idioma inglés.

Objetivos específicos:

(a) identificar los elementos asociados al idioma mapuzungun que pudiesen facilitar el proceso de aprendizaje del idioma inglés en estudiantes de origen mapuche de la Universidad Católica de Temuco que poseen nociones del mapuzungun.

(b) identificar los elementos asociados al idioma mapuzungun que pudiesen obstaculizar el proceso de aprendizaje del idioma inglés en estudiantes de origen mapuche de la Universidad Católica de Temuco que poseen nociones del mapuzungun.

\section{METODOLOGÍA}

En esta investigación se utilizó el estudio de casos múltiples, tomando en cuenta los objetivos planteados con anterioridad. El estudio de casos múltiples busca la comprensión de un fenómeno, lo cual permite descubrir e interpretar la realidad de acuerdo a la información entregada por los participantes (Pérez, 1994). Fueron casos diferentes, los cuales tuvieron como objetivo contrastar la información de cada participante con el fin de comprender mejor el proceso de Trilingüismo existente en los estudiantes mapuche que estudian inglés.

\subsection{Participantes}

Los sujetos de investigación fueron alumnos regulares de origen mapuche que estuvieran cursando cualquier año de la carrera de Pedagogía en Inglés de la Universidad Católica de Temuco. Se entendió como estudiantes de origen mapuche, todos los sujetos que fueran descendientes directos o parientes cercanos de aquellas personas que nacieron en 


$$
\begin{gathered}
\text { Bilingüismo - Trilingüismo - Lingua franca - Mapuche - Mapuzungun - Motivación } \\
\text { Intrínseca - Motivación Extrínseca - Conciencia Metalingüística - Transferencia } \\
\text { Lingüística }
\end{gathered}
$$

comunidades mapuche (Peláez, 2002). Cabe señalar que todos los participantes que decidieron colaborar con la investigación pertenecen a la carrera de Pedagogía en Inglés.

\subsection{Diseño}

Se utilizó un diseño evolutivo transversal, es decir, una serie de entrevistas, las cuales desde la segunda entrevista en adelante los datos de todos los entrevistados fueron compartidos para enfocarse finalmente en los datos más relevantes y repetitivos, los cuales se utilizaron para planificar la entrevista siguiente. Por otra parte, el carácter de transversal del diseño quiere decir que durante cada entrevista se consideró cualquier temática expresada por los participantes que pudiese aportar algo a la investigación. Este diseño fue aplicado a seis participantes de forma independiente evaluados en un tiempo único.

\subsection{Instrumentos}

Se utilizaron entrevistas en profundidad realizadas a los seis participantes en tiempos y espacios diferentes. Las entrevistas fueron todas grabadas, para posteriormente ser transcritas y analizadas según los objetivos planteados en la investigación. Pérez (1994), señala que a pesar que en los propósitos de la investigación gobiernen las preguntas a formular, su contenido, declaración y secuencia están en manos del entrevistador.

Fueron tres entrevistas en profundidad las que se realizaron a cada uno de los seis participantes, con el motivo de obtener el máximo de información que se requiere para lograr una investigación más profunda y confiable.

\subsection{Procedimiento}

Las entrevistas se guiaron de acuerdo a dos grandes ejes temáticos: la influencia del factor externo y el factor interno en el aprendizaje del idioma inglés, ellos son:

\section{Factores externos:}

a) El entorno socio-cultural y su influencia en la determinación de estudiar el idioma inglés

$>$ Entorno sociocultural, cultural, educativo

Este tema se trató en distintos niveles, se guió el relato del entrevistado de tal forma que él contara sus experiencias con su entorno de manera extensa. Finalmente, en esta parte de la entrevista se trató el tema familiar, en cuanto a las relaciones familiares, la relación de la familia y comunidad con las costumbres y tradiciones y en qué nivel han estado estas presentes en su vida.

$>$ Entorno físico más cercano

En

esta parte de la entrevista se incentivó al entrevistado a que entregara información acerca de su relación con el entorno físico donde creció y se encuentra su comunidad.

> Comunidad y gente no mapuche. Continuando con la entrevista, se guió el relato del entrevistado de tal manera que este pasara de hablar del lugar donde vive o vivió, a hablar de la gente que allí se encuentra y con la que él se ha relacionado. 


\section{Bilingüismo - Trilingüismo - Lingua franca - Mapuche - Mapuzungun - Motivación \\ Intrínseca - Motivación Extrínseca - Conciencia Metalingüística - Transferencia Lingüística}

> Familia, costumbres y tradiciones. Se guió el relato hacia la relación del entrevistado como mapuche con su cultura y la relación de su familia con la cultura, las costumbres y tradiciones.

b) Motivación Extrínseca

Se pretendió lograr que el participante formulara afirmaciones acerca de la motivación externa que tuvo, ya sea del ambiente escolar, algún miembro de la familia, o personas de su entorno cercano, que lo condujo a interesarse por aprender el idioma mapuzungun, el idioma inglés y/o por aprender de la cultura y la tradición mapuche.

\section{Factores internos:}

a) Conocimientos lingüísticos previos (Transferencia Linqüística, Conciencia Metalingüística)

Transferencia Lingüística

Este punto de la entrevista se basó principalmente en obtener información por parte del entrevistado respecto a su experiencia en el aprendizaje del idioma inglés durante su formación académica.

$>$ Conciencia Metalingüística

Este punto de la entrevista se basó en obtener información acerca de cómo los participantes creen que el conocimiento de su lengua materna (español), más sus nociones de una segunda lengua (mapuzungun) han influido en la adquisición de una tercera lengua (inglés): Español - Mapuzungun - Inglés.

b) Motivación Intrínseca

Se pretendió lograr que el participante formulara afirmaciones acerca de su propio interés y eventos que lo motivaron ya sea por aprender el idioma mapuche.

mapuzungun, el idioma inglés, y/o por aprender de la cultura y la tradición

\subsection{Análisis}

La información se analizó por medio de la clasificación de los datos, de acuerdo a los diferentes temas considerados dentro de la investigación, con el fin de buscar temáticas que fueran repetitivas y que pudieran ser profundizadas en las siguientes entrevistas. Las temáticas se categorizaron de acuerdo a los núcleos temáticos previamente establecidos y emergentes. La información entregada por los participantes se analizó utilizando la triangulación de datos. Según Denzin (1978), este método de análisis de datos permite contrastar los datos obtenidos, así como también obtener otros datos que no han sido encontrados en el primer análisis de la investigación.

Se utilizó la aplicación Excel perteneciente al Software Microsoft Office, versión 2007 para analizar los datos y encontrar la información que pudiera ser relevante para la investigación. 


$$
\begin{gathered}
\text { Bilingüismo - Trilingüismo - Lingua franca - Mapuche - Mapuzungun - Motivación } \\
\text { Intrínseca - Motivación Extrínseca - Conciencia Metalingüística - Transferencia } \\
\text { Lingüística }
\end{gathered}
$$

\section{RESULTADOS}

Luego de realizar las entrevistas semi-estructuradas realizadas a los seis participantes, se procedió a su análisis de acuerdo a lo planificado.

Los siguientes son los nombres otorgados a cada participante para resguardar sus identidades: Participante N01 "Lafquen"; Participante N02 "Millaray"; Participante N03 "Nahuel"; Participante N04 "Rayen"; Participante N05 "Sayen"; Participante N06 "Küyen".

Se analizaron las entrevistas, según las áreas preestablecidas identificando elementos facilitadores y obstaculizadores vinculados a la situación de Trilingüísmo de los participantes, los que a su vez se agruparon en factores internos y externos.

Luego de una primera ronda de análisis de las primeras entrevistas, dentro de los factores identificados como externos, se establecieron los siguientes subtemas de análisis: el entorno socio-cultural (cultural y educativo), y su influencia en la determinación de estudiar el idioma inglés, y la Motivación Extrínseca. Dentro de los elementos clasificados como factores internos, se identificaron las siguientes categorías de análisis: conocimientos lingüísticos previos (Transferencia Lingüística, Conciencia Metalingüística), y Motivación Intrínseca.

\section{Caso No1}

Análisis de la muestra correspondiente a las entrevistas realizadas al participante "Lafquen"

El participante tiene veintidós años de edad, es de sexo masculino y estudiante de cuarto año de la carrera de Pedagogía en Inglés en la Universidad Católica de Temuco.

\subsection{Relación con la cultura}

En cuanto a la relación del participante con la cultura mapuche, él señala que se siente parte de la cultura, y que el hecho de participar en celebraciones mapuche le permite acercarse mucho más a sus raíces:

"Ehh... la cultura Mapuche igual es... es como... ehh, buta no sé cómo decirlo pero igual es más como, más como piola, no sé. Es como..." 
Bilingüismo - Trilingüismo - Lingua franca - Mapuche - Mapuzungun - Motivación

Intrínseca - Motivación Extrínseca - Conciencia Metalingüística - Transferencia

Lingüística

\subsection{Relación con el mapuzungun}

Lafquen señala que no tuvo ninguna influencia de su entorno cercano en donde pudiese aprender el mapuzungun. Aquello se relaciona con su Motivación Extrínseca correspondiente al factor externo en el aprendizaje del idioma inglés:

"En realidad no. Lo que pasa es que estudié en Coronel, pero en un Liceo donde no era... como... no era rural, y así que no tenían cursos especiales de mapuzungun, entonces no tuve relación con el mapuzungun en la escuela" (Entrevista 2).

\subsection{Relación con el inglés}

Con respecto a la relación del participante con el idioma inglés, se puede señalar que él siempre tuvo la facilidad de aprender el idioma debido a su interés, por lo que nunca tuvo problemas por aprender inglés en la escuela:

"Ehh... inglés... igual siempre me gustó desde la básica. Ehh... no... no se me hacía muy difícil en comparación a lo que era con mis compañeros. Y de ahí parte todo, desde la básica" (Entrevista 1).

\subsection{Relación mapuzungun-inglés}

Lafquen menciona en la entrevista que el tener nociones del idioma mapuzungun podría obstaculizar el aprendizaje del inglés, debido a que el mapuzungun posee fonemas que no existen en el idioma inglés, porque los dos idiomas tienen raíces lingüísticas distintas:

"Ehhh... Son pocas yo creo las similitudes. Hay fonemas que (...) que no existen ehh, en una, y en la otra sí. Fonemas de una lengua que no existen en la otra" (Entrevista 1).

\subsection{Trilingüísmo}

En cuanto al tema del Trilingüísmo, Lafquen señala que en el caso de estos tres idiomas, no existe una mayor relación entre sí, ya que los tres idiomas provienen de raíces lingüísticas distintas. No obstante, agrega que en la pronunciación de sólo algunas palabras, se asemejan los tres idiomas, por lo que aquello podría ser una relación de orden fonético:

"Bueno, yo creo que ninguno de ellos se parece tanto, porque los tres vienen de partes distintas, así que es lo que pienso... ahora igual tienen cosas en común, como por ejemplo... por ejemplo el español se parece al inglés en la pronunciación de algunas letras, al igual que el mapuzungun con el inglés... ahí como que se parecen los tres juntos. Pero, como te digo, en lo demás, son muy distintos los idiomas" (Entrevista 2). 


\section{Bilingüismo - Trilingüismo - Lingua franca - Mapuche - Mapuzungun - Motivación \\ Intrínseca - Motivación Extrínseca - Conciencia Metalingüística - Transferencia \\ Lingüística}

\section{Conclusiones}

\section{Elementos Facilitadores}

- Se identifica como un posible elemento facilitador en el aprendizaje del inglés la motivación, ya que Lafquen señala que uno de sus propósitos de aprender inglés es conocer más de otras culturas, por lo tanto, se entiende que es un factor motivante el aprender más idiomas.

- Otro posible elemento facilitador del aprendizaje del inglés es el interés por aprenderlo con el fin de utilizarlo como una herramienta para crear proyectos en donde pueda utilizar los dos idiomas y enseñar al mundo su cultura mapuche.

- Respecto a la relación mapuzungun-inglés, Lafquen enfatiza que en los dos idiomas podrían facilitarse entre sí por varias razones, estas son:

1- ambos idiomas se asemejan en algunas pronunciaciones de consonantes específicos.

2- existe más confianza para una persona cuando ya se ha adquirido más de un idioma (factor motivacional).

3- el idioma mapuzungun posee estructuras gramaticales y formaciones de oraciones que coinciden con algunas estructuras del idioma inglés.

4- el tener nociones del idioma mapuzungun ayuda a ampliar el vocabulario al momento de comenzar a aprender inglés.

- Con respecto al Trilingüísmo, Lafquen señala que el tener conocimiento de tres idiomas, en este caso, hablar español, hablar inglés y tener conocimientos del idioma mapuzungun, es muy valorable y menciona que mientras más idiomas puede hablar, más valor social adquiere.

\section{Elementos Obstaculizadores}

- En cuanto a la relación mapuzungun-inglés, Lafquen menciona que el tener nociones del idioma mapuzungun podría obstaculizar el aprendizaje del inglés, debido a que el mapuzungun posee fonemas que no existen en el idioma inglés.

- En cuanto al tema del Trilingüísmo, Lafquen señala que en el caso de estos tres idiomas, no existe una mayor relación entre sí, ya que los tres idiomas provienen de raíces lingüísticas distintas.

\section{Caso No2}

Análisis de la muestra correspondiente a las entrevistas realizadas al participante "Millaray"

El participante es de sexo femenino, tiene veintidós años de edad y actualmente está cursando el quinto año de Pedagogía en Inglés en la Universidad Católica de Temuco.

\subsection{Origen étnico}

Millaray comienza la primera entrevista refiriéndose a su lugar de procedencia. En este sentido da a conocer lo siguiente: 
Bilingüismo - Trilingüismo - Lingua franca - Mapuche - Mapuzungun - Motivación

Intrínseca - Motivación Extrínseca - Conciencia Metalingüística - Transferencia

Lingüística

"Bueno el área donde yo nací es un sector rural donde vive... bueno el mayor porcentaje de la gente que vive ahí es mapuche, y... está bien alejado de la ciudad (...)comunidad indígena "Domingo Pichinao" que se llama. "(Entrevista No1)

\subsection{Antecedente familiar}

Sobre el antecedente familiar de la participante en relación al mapuzungun, ella da a conocer lo siguiente:

"Ya, en relación al lenguaje... a ver... obviamente en mi casa todos hablaban español y también mapuzungun (...) mi bisabuela con mi tío discutían en mapuzungun (...)" (Entrevista No1)

la participante manifestó que toda su familia hablaba mapuzungun, pero lo hablaban sólo en ciertas ocasiones (discusiones y conversaciones entre su bisabuela y tío).

\subsection{Relación con el mapuzungun}

Millaray comentó lo siguiente sobre su acceso al mapuzungun:

"Entonces yo tenía acceso al idioma mapuzungun porque... en los guillatunes, en la rogativas que hacían porque yo asistía a todas. Tenía que asistir y todas se hacían en mapuzungun. " (Entrevista No1)

De la cita anterior, se desprende que Millaray tuvo acceso al mapuzungun mediante la participación en "guillatunes" y "rogativas". Además, ella señaló que un miembro de su familia, que vive en su casa, habla el mapuzungun, la cual actualmente constituye su única conexión con tal idioma.

"(...) cuando voy a mi casa a veces mi tío habla mapuzungun pero no conmigo sino que habla solo o dice cosas en mapuzungun y por ahora ese el acceso que tengo, no es como antes cuando yo era niña." (Entrevista No1)

\subsection{Interés por el idioma inglés}

Millaray tuvo interés por aprender inglés:

"(...) amiga que tenía amigos gringos entonces yo siempre iba a su casa y los gringos siempre estaban ahí (...) y... ahí siempre los escuchaba a ellos hablar inglés y la curiosidad me llevo a estudiar inglés y a estar interesada." (Entrevista No1)

El interés de Millaray por el inglés se puede señalar que viene de un factor interno, específicamente la Motivación Intrínseca. La curiosidad por saber qué hablaban los hablantes nativos con quienes tuvo contacto en su infancia puede haber gatillado a estar interesada en el inglés y luego estudiar dicho idioma. 
Bilingüismo - Trilingüismo - Lingua franca - Mapuche - Mapuzungun - Motivación

Intrínseca - Motivación Extrínseca - Conciencia Metalingüística - Transferencia

Lingüística

\subsection{Relación con el idioma inglés en el sistema educativo}

La participante mencionó que tuvo acceso al inglés desde 5to básico.

"Después en el colegio cuando estaba en 5to no me acuerdo la edad pero desde 5to hacia adelante. En básica de 5to a 8vo ahí tuve acceso a inglés. " (Entrevista No2) siguiente:

Respecto a su relación con el inglés en la enseñanza media, Millaray manifestó lo

"(...) media estudié en un colegio de hotelería y turismo así que tenia... (...) 5 (horas) de inglés y todas las pruebas que nos hacían eran 2 pruebas escritas en el año y las demás todas orales asi que "speaking" era harto, hacíamos "role play" por ejemplo." (Entrevista No2)

\subsection{Relación mapuzungun-inglés}

Millaray comentó lo siguiente sobre cómo el mapuzungun le habría ayudado a aprender el inglés:

"Yo creo que lo que me ha facilitado el aprendizaje del inglés es el conocimiento del mapuzungun, principalmente algunos sonidos como la r, la $\theta$ (esa de think), también algunos sonidos con vocales como la a (shua) y algunas vocales que están juntas y no es fácil pronunciarlas. " (Entrevista No1)

De las citas anteriores se desprende explícitamente que el mapuzungun ha facilitado a Millaray el proceso de internalización de distintos sonidos, fonemas, pronunciación y estructuras del inglés.

\subsection{Trilingüísmo}

Millaray comentó lo siguiente sobre la relación entre el español, mapuzungun e inglés:

"Esto fue porque esa "r" yo la escuchaba y la podía pronunciar bien, de hecho, cuando yo fui... antes de aprender a hablar español, o sea antes de poder hablar bien el español yo pronunciaba la " $r$ " (tono suave) y no podía decir la... no se po'... por ejemplo "redondo" (letra "r" en tono fuerte y marcado) y decía "redondo" (letra "r" con tono suave) es que siempre escuchaba esa " $r$ " en los guillatunes y aparte que mi tío igual hablaba mapuzungun así como solo." (Entrevista No1)

La participante da a conocer como el mapuzungun tuvo influencia en la pronunciación del inglés e incluso en el español. Se puede deducir, en base a las palabras de Millaray, que el tener nociones de mapuzungun le facilitó la pronunciación del inglés y además, del español. Por tanto, las nociones actuaron como elementos facilitadores.

\section{Conclusiones}


Bilingüismo - Trilingüismo - Lingua franca - Mapuche - Mapuzungun - Motivación

Intrínseca - Motivación Extrínseca - Conciencia Metalingüística - Transferencia

Lingüística

Elementos facilitadores

- Las nociones del mapuzungun ayudaron y facilitaron a la entrevistada el proceso de aprendizaje del inglés. Específicamente facilitaron la internalización y aprendizaje de ciertos patrones relacionados a la fonética, pronunciación y vocabulario en inglés. Todo esto debido a la similitud existe entre algunos sonidos del mapuzungun y el inglés.

- De las entrevistas analizadas a Millaray no se identificaron elementos, asociados al mapuzungun, que pudiesen haber obstaculizado el aprendizaje del inglés.

\section{Caso N03}

Análisis de la muestra correspondiente a las entrevistas realizadas al participante "Nahuel"

El siguiente es un análisis en profundidad de las tres entrevistas realizadas a un alumno de pedagogía en inglés al que llamaremos por motivos de confidencialidad "Nahuel".

\subsection{Origen étnico}

El origen mapuche del participante es manifestado por este en forma clara como se observa en la siguiente cita:

"En mi infancia relacionada con la cultura, yo cuando era chico, vivía acá en...nací acá en Lautaro, entonces sin haber hospital y todo el asunto" (Entrevista uno)

\subsection{Conciencia metalingüística}

De las afirmaciones de Nahuel de la relación entre mapuzungun y el inglés se desprenden elementos que se enmarcan dentro del proceso cognitivo que ocurre al aprender una lengua conocido como conciencia metalingüística.

\footnotetext{
"Si, en el sentido que uno te ayuda a abrir un poco más la mente y a entender un poco más el otro. A mi me gustan harto los idiomas, no tanto en aprender el vocabulario sino que en entender el transfondo y la parte cultural, no tanto lo practico del asunto." (Entrevista 2)
}

Nahuel plantea que el mapuzungun como idioma en especial no ayuda mucho a la parte gramatical y más práctica en el aprendizaje del idioma inglés. Sin embargo, también menciona que el hecho de estar consciente del aprendizaje de un idioma y a la vez estar consciente de cuáles son los diferentes componentes que tienen los idiomas, ayuda a entender un segundo o tercer idioma con mayor facilidad, sobre todo al momento de dejar de lado tu lengua madre para adentrarte en otra lengua.

\subsection{Conciencia Metalingüística y Trilingüísmo}

Nahuel cuenta que no solamente el hecho de estar consciente de los procesos cognitivos que están ligados al aprendizaje de un idioma ayudan a la adquisición de un segundo, sino que va 


\title{
Bilingüismo - Trilingüismo - Lingua franca - Mapuche - Mapuzungun - Motivación \\ Intrínseca - Motivación Extrínseca - Conciencia Metalingüística - Transferencia \\ Lingüística
}

más allá y plantea que también sucede lo mismo con la adquisición de un tercer idioma y una retroalimentación en el aprendizaje entre estos.

Nahuel plantea que al tener una conciencia lingüística del proceso de adquisición de los idiomas se hace más fácil su comprensión y se produce un feedback entre los idiomas que ayuda a comprender como estos funcionan. Este proceso mental entonces, según Nahuel, lo ha ayudado a aprender una segunda y tercera lengua de manera más amigable y rápida.

\subsection{Transferencia lingüística}

De las entrevistas se desprenden comentarios que apuntan a el proceso de Transferencia Lingüística, aunque no le da un valor muy importante a entre la relación mapuzungun-inglés.

\begin{abstract}
"No, totalmente distinto, igual hay cosas que se parecen como la " $R$ " en mapuzungun y la " $R$ " en inglés, yo me atrevería a decir son iguales y la otra es que en el mapuzungun en la parte fonética hay una especie de " $\partial$ " que igual se parece a la del inglés y también esta a " $Z$ " del mapuzungun que también la tiene el inglés, pero por otra parte esta la sexta vocal del mapuzungun que no la tiene el inglés y tampoco el castellano, se articula de una manera especial, se puede decir que tiene cosas parecidas, pero no es mucho. " (Entrevista 2)
\end{abstract}

\section{Conclusiones}

\section{Elementos facilitadores}

Las entrevistas realizadas a Nahuel revelaron dos factores relacionados al idioma mapuzungun que pueden influir en el aprendizaje del idioma mapuzungun.

El primer factor guarda relación con la cultura mapuche, específicamente la motivación que creó en Nahuel su profesora de inglés que también era mapuche, ella lo incentivó de manera extrínseca e intrínseca, ya que la imagen que proyecto lo motivó aparte de la propia motivación más explícita realizada por la profesora.

Nahuel plantea que el estar consciente de los procesos mentales que ocurren al aprender un segundo o tercer idioma, hacen que sea más fácil la adquisición y comprensión de estos. La habilidad de desprenderse de la lengua madre se produce de manera más rápida al tener conciencia de cómo funcionan los idiomas y se realiza a la vez un feedback entre los idiomas adquiridos. Este feedback a la vez se puede enmarcar dentro del proceso de Transferencia Lingüística, ya que de las palabras de Nahuel podemos inferir que la Transferencia Lingüística y la Conciencia Metalingüística no son procesos necesariamente que ocurran de manera separada en el aprendizaje del inglés.

\section{Elementos Obstaculizadores}

El único factor ligado al idioma mapuzungun que parece afectar de manera negativa el aprendizaje del idioma inglés, tiene que ver con la relación que se ha producido entre la cultura mapuche y la cultura occidental en la vida de Nahuel. La dicotomía que se ha producido entre estas cultura durante el proceso educativo parece haber distanciado a Nahuel del idioma inglés durante una parte importante de su vida. Lo que expresa más explícitamente es el perjuicio que dice haber hacia el idioma mapuzungun por el hecho de privilegiar idiomas que no pertenecen a este lugar del mundo. 
Bilingüismo - Trilingüismo - Lingua franca - Mapuche - Mapuzungun - Motivación

Intrínseca - Motivación Extrínseca - Conciencia Metalingüística - Transferencia

Lingüística

\section{Caso No4}

Análisis de la muestra correspondiente a las entrevistas realizadas al participante "Rayen"

El cuarto participante ha sido denominado como Rayen, la cual es de sexo femenino, de veinte años de edad y que actualmente cursa el segundo año de Pedagogía en Inglés en la Universidad Católica de Temuco.

\subsection{Origen étnico}

Rayen expresó lo siguiente sobre su origen étnico:

"¿Donde nací? Ehhh, mmm... acá en Temuco. Mira, mi mamá me tuvo acá en Temuco y creo que estuvimos tres años, porque después ella se casó y nos fuimos a vivir a la Argentina. Allá estuve hasta los siete años, de los 3 a los 7" (entrevista 2)

La participante manifestó que nació en la ciudad de Temuco, pero después por motivos familiares fue a vivir a otro país.

\subsection{Relación con el mapuzungun}

Rayen comentó lo siguiente sobre su relación con el mapuzungun:

"yo tengo tíos y esos tíos los van a visitar gente que habla mapudungun. Entonces yo me acuerdo que de repente vendían animales e iba gente y era típico que se hacía una comida. Entonces venía gente y la gente hablaba en mapudungun. Yo miraba así y me sorprendía y me gustaba, me gustaba como escuchar." (entrevista 2)

\subsection{Interés por el mapuzungun.}

En los siguientes datos podremos apreciar el interés que Rayen manifiesta por el idioma mapuzungun.

"me gustaba vivir en el campo y de hecho cuando yo iba al campo, me iba en esas micros rurales que hay y ahí era típico que iba la gente mapuche, hablaban mapudungun, hablaban su lengua y a mi eso siempre me despertó curiosidad" (entrevista 2)

\subsection{Inglés}

De la relación de Rayen con el inglés se identificaron los siguientes elementos: relación con el inglés e interés por el inglés y la influencia de un profesor de inglés.

\subsection{Relación con el inglés}




\section{Bilingüismo - Trilingüismo - Lingua franca - Mapuche - Mapuzungun - Motivación \\ Intrínseca - Motivación Extrínseca - Conciencia Metalingüística - Transferencia \\ Lingüística}

Rayen comentó lo siguiente sobre motivación externa que recibió por parte de un grupo de misioneros.

"yo estaba esa vez con unos chicos que son misioneros, entonces ellos me decían Rayen el inglés es súper importante, tu nos podrías ayudar " $N$ ". (entrevista 2)

\subsection{Trilingüísmo}

En esta categoría haremos alusión a la relación que nuestra participante entre mapuzungun y el inglés.

\subsection{Relación mapuzungun-inglés} el inglés

En el siguiente dato Rayen señala un factor en común que tuvo con el mapuzungun y "...Por la parte de la curiosidad puede ser que tenga alguna cercanía, pero más que
nada yo creo que a mi lo que me influenció a estudiar inglés es por lo necesario que
es"(entrevista 2)

De manera explícita, Rayen menciona que no existe una relación entre inglés y mapuzungun. La similitud entre los idiomas se reduce a la curiosidad que nuestra entrevistada tenía por las lenguas.

\section{Conclusiones}

\section{Elementos obstaculizadores}

De los elementos que podrían ser obstaculizadores, se encuentra la crítica al conflicto mapuche. Es aquí donde encontramos un factor del entorno sociocultural el cual se puede traducir en un rechazo al aprendizaje del inglés. Dentro de la entrevista de cotejo, nuestra entrevistada encuentra que es injusto el énfasis al inglés y la discriminación que existe en relación a la preservación de las tradiciones mapuches.

\section{Elementos facilitadores}

Dentro de las entrevistas realizadas no se encontraron datos que apelen posibles facilitadores. Por otra parte, podemos señalar que su interés por los idiomas se basa en una motivación interna intrínseca, por una curiosidad o interés por la comunicación. Durante la entrevista de cotejo Rayen confirmó que su interés en el inglés comenzó por curiosidad y gracias a la motivación que le brindó un grupo de misioneros cuando ella era niña. La participante descarta completamente la influencia del mapuzungun para la adquisición de una nueva lengua.

\section{Caso N05}

Análisis de la muestra correspondiente a las entrevistas realizadas al participante "Sayen" 
Bilingüismo - Trilingüismo - Lingua franca - Mapuche - Mapuzungun - Motivación

Intrínseca - Motivación Extrínseca - Conciencia Metalingüística - Transferencia

Lingüística

El quinto participante entrevistado, denominado como "Sayen" es de sexo femenino, de veinte años de edad y estudiante de segundo año de la carrera de Pedagogía en Inglés en la Universidad Católica de Temuco.

\subsection{Relación con la cultura}

Sayen manifestó que posee conocimiento sobre costumbres propias de su cultura.

"E... pero sí me manejo un poco con lo que son las costumbres... qué es lo que ellos hacen, sus rituales... cosas... cosas asi.." (Entrevista 1)

La participante manifiesta conocer aspectos de la cultura mapuche, ya que ella nombra acontecimientos y situaciones propias de la cultura de esta etnia.

\subsection{Idioma mapuzungun}

Esta categoría corresponde a comentarios realizados por Sayen sobre: su interés y relación con el mapuzungun, su antecedente familiar y el apoyo familiar.

\subsection{Relación con el idioma mapuzungun}

Sayen manifiesta lo siguiente sobre su relación con el mapuzungun.

"Claro por que por ejemplo en el colegio nosotros, por el director del colegio él sabía mapudungun, entonces como que hacia talleres. En donde nos enseñaba... un poco... mapudungun, igual entonces ahí empecé a como interesarme más de... de... del lenguaje de..." (Entrevista 1)

De la cita anterior se entiende que la participante tuvo acceso al mapuzungun a través de talleres en la enseñanza básica. Tal situación produjo que Sayen se interesara por conocer más de su idioma.

\subsection{Antecedente familiar}

Esta categoría alude a la familia de Sayen, en términos de sus conexiones con la etnia mapuche y como esa conexión le ha permitido acercarse al idioma mapuzungun. En relación a lo anterior, ella manifestó lo siguiente:

"...pero igual he tenido contacto con... con... gente... map... indígena mapuche que son la familia de mí papá. Con ellos como que aprendí un poco... entre comillas (Entrevistada gesticula con sus manos) e... un poco e... el len... el lenguaje que ellos... que ellos utilizan". (Entrevista 1)

\subsection{Interés por el idioma inglés}

En cuanto al interés por el inglés, Sayen manifestó lo siguiente: 


$$
\begin{gathered}
\text { Bilingüismo - Trilingüismo - Lingua franca - Mapuche - Mapuzungun - Motivación } \\
\text { Intrínseca - Motivación Extrínseca - Conciencia Metalingüística - Transferencia } \\
\text { Lingüística }
\end{gathered}
$$

"El idioma, por que me gustó mucho... desde chica me... me gustó, me llamó la
atención. Así que esta fue mí única opción la primera la única (...)"(Entrevista 2).

De las citas expuestas anteriormente se desprende que Sayen desde pequeña manifestó curiosidad por el inglés. A su vez tal curiosidad se expresaba en conocer lo que personas hablantes de la lengua inglesa expresaban mediante el uso del tal idioma. Además, ella señala el inglés fue su primera opción para estudiar en la universidad.

\subsection{Relación inglés-mapuzungun}

En cuanto a la relación entre el mapuzungun y el inglés, Sayen manifestó lo siguiente:

"Em... (...) mira yo se un poco de mapudungún y se que las estructuras van
cambiando y la cosa así. Entonces, como que en el inglés a veces son como
medias parecidas, cosas asi." (Entrevista 3)

De acuerdo a la cita anterior, el conocimiento que Sayen posee del mapuzungun le permite darse cuenta que ocurren cambios en las estructuras de los idiomas. Además, ella señala que las estructuras del mapuzungun se asemejan en alguna medida a las estructuras del inglés.

\section{Conclusiones}

Elementos facilitadores

Las nociones del mapuzungun habrían ayudado a la entrevistada en el aprendizaje del inglés, específicamente en la pronunciación y adquisición de palabras en inglés. Además, ella señaló que su conocimiento del mapuzungun le permitió asociar cambios en las estructuras en tal idioma al inglés. Finalmente, las nociones habrían permitido que Sayen enfrentara con mayor confianza el aprendizaje de un nuevo idioma (inglés).

\section{Elementos obstaculizadores}

De las entrevistas emergió un aspecto asociado el mapuzungun que podría obstaculizar el aprendizaje del inglés, el cual se relaciona al entorno de aprendizaje del mapuzungun, el que según la entrevistada es completamente distinto al del inglés.

\section{Caso $\mathrm{N}^{\circ} 6$}

\section{Análisis de la muestra correspondiente a las entrevistas realizadas al participante} "Küyen"

El participante, "Küyen", de sexo masculino y de diecinueve años de edad se encuentra cursando el segundo año de Pedagogía en Inglés en la Universidad Católica de Temuco. Las entrevistas, hechas a Küyen, ratifican su origen étnico mapuche, el interés por su cultura y por el mapuzungun, así como su relación e interés por el inglés.

\subsection{Relación con la cultura}




\section{Bilingüismo - Trilingüismo - Lingua franca - Mapuche - Mapuzungun - Motivación \\ Intrínseca - Motivación Extrínseca - Conciencia Metalingüística - Transferencia Lingüística}

En relación a ceremonias o costumbres de la cultura mapuche, Küyen no era tan participe, a pesar de que asistía, esto en el marco que hace una comparación entre sus compañeros de colegio y él.

"... habian compañeros que eran de campo, más que yo, más al campo

tenían más costumbres de los mapuches, pero yo no era tan cercano a

las costumbres." (Entrevista $N^{\circ} 1$ ).

\subsubsection{Mapuzungun}

En relación a lo expresado por Küyen acerca del mapuzungun, se encuentra lo siguiente a destacar.

\subsubsection{Relación con el idioma mapuzungun}

La relación del participante con el mapuzungun en el ambiente familiar se manifiesta en esta cita:

"En mi familia hay varias personas, eh integrantes que hablan mapudungun, principalmente, mi abuela, mi tía abuela y bisabuela... pero como que no lo hablaban tanto, sino que con el tiempo se fue perdiendo porque no tenían con quien hablar entonces me enseñaron algunas palabras y cosas así, ahora con el tiempo yo como... que he olvidado algunas palabras". (Entrevista $N^{\circ} 1$ ).

\subsubsection{Interés por el idioma mapuzungun}

El participante tuvo acceso al mapuzungun a través de la interacción con un miembro de su familia. Producto de esto, habrían hecho que él se interesara por conocer más de su idioma para poder establecer un grado más alto de comunicación con ese pariente.

\subsubsection{Relación mapuzungun-inglés}

Esta categoría emergió de los comentarios que Küyen realizó sobre similitudes y diferencias, que a su parecer existían entre los dos idiomas.

\section{- $\quad$ Similitudes}

De acuerdo a Küyen, una de las similitudes es que él tuvo una relación muy cercana con los dos idiomas y a estos dos los consideraría como L2, idiomas adquiridos como segunda lengua.

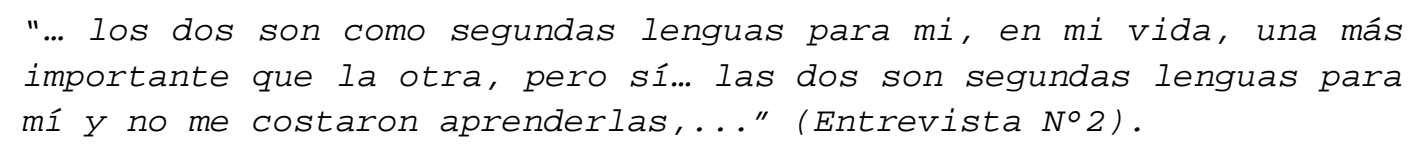

Diferencias 


\section{Bilingüismo - Trilingüismo - Lingua franca - Mapuche - Mapuzungun - Motivación \\ Intrínseca - Motivación Extrínseca - Conciencia Metalingüística - Transferencia Lingüística} inglés.

En cuanto a las opiniones de Küyen, no existirían diferencias entre el mapudungun e

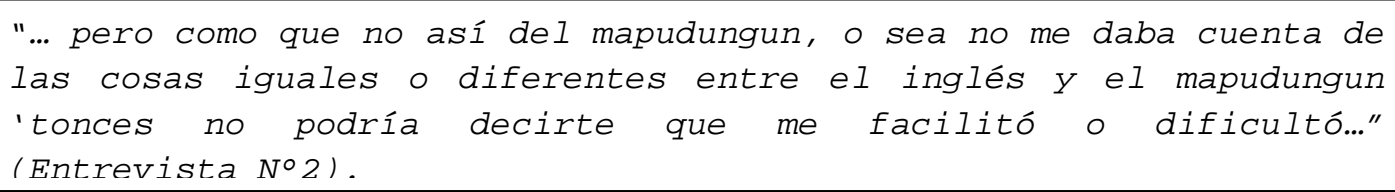

\section{Conclusiones}

\section{Elementos facilitadores}

- El participante se interesa en el mapuzungun con la intención de poder comunicarse con un familiar, del mismo modo, cuando tiene contacto con personas de habla inglesa, él se interesa por sostener un grado de comunicación con estas personas, produciéndose una Motivación Intrínseca, de orden interno. Esto sería un elemento asociado al mapuzungun que facilitaría el aprendizaje del inglés, porque en los dos casos jugó un rol primordial la motivación, ya que llevó a Küyen a interesarse por estos dos idiomas, y así aprenderlos para poder comunicarse con otras personas. $Y$ en el caso del inglés a estudiarlo en una carrera de pregrado. Sin embargo, se puede afirmar, que el interés por aprender mapuzungun no era tan significativo, como en el inglés.

- Kúyen señala que no le fue tan difícil el aprendizaje del inglés, puesto que contaba con nociones del mapuzungun, y a la vez, estaba consciente de que habían ciertos elementos del mapuzungun que se asimilaban o eran idénticos en el inglés. Estos elementos son de orden fonético. Lo dicho por el participante tiene que ver con un factor interno relacionado con la Conciencia Metalingüística. Lo cual sería un elemento asociado al mapuzungun que facilitaría el aprendizaje del inglés, ya que está consciente del hecho que existen fonemas idénticos en los dos idiomas, y conocer primero esos sonidos en el mapuzungun lo habría llevado a identificarlos en el inglés con facilidad, y así aprender otras características del idioma.

\section{Elementos obstaculizadores}

- No obstante, Küyen señala un factor externo, relacionado con el contexto sociocultural y escolar que se asocia al mapuzungun, el cual obstaculizaría el aprendizaje del inglés, debido a que el proceso de enseñanza aprendizaje del inglés es obligatorio en todo nuestro país, mientras que el mapuzungun no lo es. Esto impediría el poseer conocimiento, nociones del mapuzungun en la educación formal, y esto incidiría en no poder acceder a elementos de este último, los cuales facilitarían el aprendizaje del inglés o cualquier otro idioma.

\section{DISCUSIÓN}

El objetivo de esta investigación es determinar cuáles son los elementos asociados al idioma mapuzungun que facilitan u obstaculizan el aprendizaje del idioma inglés. En este sentido, son diversos los factores que influyen en el aprendizaje del inglés como un tercer idioma. 


\section{Bilingüismo - Trilingüismo - Lingua franca - Mapuche - Mapuzungun - Motivación \\ Intrínseca - Motivación Extrínseca - Conciencia Metalingüística - Transferencia \\ Lingüística}

En relación a los elementos ligados a la cultura mapuche, se encontraron elementos que pudieron haber facilitado y obstaculizado el aprendizaje del inglés. Respecto a los elementos facilitadores asociados a esta subcategoría, surgieron dos factores que motivaron de manera intrínseca y extrínseca a dos de los participantes.

Uno de los participantes expresó su interés por trabajar en el área del etnoturismo y mostrar la cultura a extranjeros (véase análisis 5.1.1.2). De acuerdo a Romero (2009), la motivación intrínseca se asocia a factores internos de la persona, por ejemplo gustos o intereses por alguna situación, experiencia o actividad en particular. Por lo tanto, se puede afirmar que el móvil de este participante guarda relación con el interés por utilizar el idioma inglés como una herramienta para dar a conocer al mundo su propia cultura.

El segundo factor está asociado a la relación de uno de los entrevistados con su profesora de inglés quien también era de origen mapuche (véase análisis 5.3.1.2). Ella lo incentivó de manera explícita en el aprendizaje del inglés. Según Romero (2009), la motivación extrínseca se encuentra en factores externos. La persona no se siente interesada por la situación, experiencia o actividad en sí, sino que busca conseguir otros fines (un título, un trabajo, reconocimiento social, entre otros). Este tipo de motivación provocada por la profesora fue un factor que influyó de manera positiva en el proceso de aprendizaje del inglés. Además, él vio en ella un modelo positivo y sin prejuicios sobre la cultura occidental y el aprendizaje del inglés (véase análisis 5.3.1.2).

En relación a los elementos asociados a la cultura mapuche que pudiesen haber obstaculizado el aprendizaje del inglés, un participante asoció la cultura mapuche con la cultura occidental.

Tal participante expresó un cierto rencor hacia la cultura occidental. El señaló que las tradiciones fueron impuestas de manera violenta por los colonizadores (véase análisis 5.3.1.3). En una segunda entrevista señala que los idiomas español e inglés se enseñan en desmedro del mapuzungun, y que por lo tanto, el inglés y el español, al ser idiomas extranjeros, deberían ser enseñados en segundo lugar de importancia. Respecto a la relación mapuzungun-inglés, surgieron datos que pudieron haber facilitado, así como también obstaculizado el aprendizaje del inglés.

En este sentido, seis participantes señalaron que los conocimientos del idioma mapuzungun influyeron de alguna manera al aprendizaje del inglés, proceso que se denomina Transferencia Lingüística. Según Otto (2007), la Transferencia Lingüística, sea esta positiva o negativa, puede verse reflejada en diferentes niveles del lenguaje: en el fónico, en el léxico, en el semántico, en el morfosintáctico, en el sociocultural, en el sociolingüístico, etc., tanto en la producción como en la percepción, tanto en la lengua oral como en la lengua escrita. Por tanto, cuatro participantes manifestaron que poseer nociones de mapuzungun facilitó la adquisición del inglés. Esto ocurre debido a la similitud en cuanto a la fonética y a las estructuras gramaticales que existe entre dichos idiomas. De acuerdo a lo señalado por Ludmila (2005), durante el proceso de adquisición de una segunda lengua, el conocimiento que se tiene de la lengua nativa (L1) en el proceso de adquisición de una segunda lengua (L2) puede realmente lograr tener un efecto "facilitador o de inhibición" en el progreso del aprendiz de dominar un nuevo idioma. Dado al número de participantes que manifestaron la influencia positiva de un idioma hacia el otro, se puede indicar que es un factor no menor en el proceso de aprendizaje del inglés por parte de alumnos que poseen nociones del mapuzungun. No obstante, de los comentarios realizados por un participante, se encontró que la relación 


$$
\begin{gathered}
\text { Bilingüismo - Trilingüismo - Lingua franca - Mapuche - Mapuzungun - Motivación } \\
\text { Intrínseca - Motivación Extrínseca - Conciencia Metalingüística - Transferencia } \\
\text { Lingüística }
\end{gathered}
$$

mapuzungun-inglés aludía a una Transferencia Lingüística que pudiese haber obstaculizado el aprendizaje del idioma inglés (véase análisis 5.1.4). Tal obstaculización se manifestaría debido a diferencias entre fonemas existentes entre el idioma mapuzungun e inglés. Ludmila (2005), señala que existe "Transferencia Lingüística negativa" cuando la lengua madre actúa como "inhibidor" en el aprendizaje de la L2. Además, esta situación se asemeja a lo que Carabantes, Castillo y Pasten (2006) plantean, según estos autores, se produce un mayor grado de dificultad en el aprendizaje de la L2 cuando esta y la L1 presentan mayores diferencias. En cuanto a la relación entre el idioma español, mapuzungun e inglés, se encontró un caso en el que se manifestaba que los tres idiomas provienen de raíces lingüísticas distintas (véase análisis 5.1.5.). De acuerdo a Carabantes, Castillo y Pasten (2006), la existencia diferencias entre los idiomas que maneja el aprendiz y el que él quiere aprender le producen una mayor dificultad en el proceso de adquisición de la nueva lengua. Sumado a lo anterior, Ludmila (2005) establece que la lengua materna puede lograr tener un efecto "facilitador o de inhibición" en el progreso del aprendiz en dominar una nueva lengua. Este caso específicamente, al existir diferencias entre los tres idiomas se produciría lo que Ludmila (2005) denomina "Transferencia Lingüística negativa".

En relación a la Conciencia Metalingüística, se encontró que sólo un participante estaba consciente de los procesos mentales que ocurren al momento de aprender un idioma. Según Lasagabaster (1999), el desarrollo de la competencia lingüística en dos lenguas puede resultar en niveles más altos de la Conciencia Metalingüística (CM) y la facilitación del aprendizaje de una tercera lengua. Este participante manifestó que la comprensión de un segundo o tercer idioma se realiza con mayor facilidad luego de haber generado la habilidad de desprenderse de su lengua madre y adentrarse a otra lengua en un proceso de comprensión del idioma más que una mera traducción (véase análisis 5.3.2.1.1). Bialystok (2007) postuló que la Conciencia Metalingüística se define como el explicitar de manera consciente la forma lingüística y estructural del lenguaje para considerar como ellas se relacionan y producen el significado subyacente de las oraciones. Es así como el participante ha manifestado que esta comprensión de los significados y de las estructuras del idioma mapuzungun han favorecido en el aprendizaje del idioma inglés, produciéndose un proceso más amigable e interesante, incluso hay una retroalimentación entre los idiomas a nivel fonológico y semántico (véase análisis 5.3.2.1.2).

Finalmente, los hallazgos permiten establecer que el elemento asociado al mapuzungun que facilitó el aprendizaje del inglés en los participantes es la Transferencia Lingüística, ya que este factor se identificó en cuatro de los seis casos. Ellos señalaron que al haber elementos parecidos entre los dos idiomas, aquello facilitó el proceso de aprendizaje del inglés.

Igualmente, hubo otros factores que influyeron positivamente en el proceso de aprendizaje del inglés, tales como la Conciencia Metalingüística y factores socioculturales, los cuales se hallaron en tan sólo un caso.

En cuanto a los factores asociados al mapuzungun que obstaculizaron el aprendizaje del inglés, se encontró que en la relación entre la cultura mapuche y occidental, existen elementos que obstaculizaron el proceso de aprendizaje en un participante. Por otra parte, se presentó el caso en el cual la diferencia de origen lingüístico del español, mapuzungun e inglés resultó ser un elemento obstaculizador en el aprendizaje del inglés. 


$$
\begin{gathered}
\text { Bilingüismo - Trilingüismo - Lingua franca - Mapuche - Mapuzungun - Motivación } \\
\text { Intrínseca - Motivación Extrínseca - Conciencia Metalingüística - Transferencia } \\
\text { Lingüística }
\end{gathered}
$$

\section{REFERENCIAS}

Bialystok, E. (2007). Acquisition of Literacy in Bilingual Children: A Framework for Research. Language Learning. Malden: Blackwell Publishing.

Carabantes, L; Castillo, M; Pasten, M. (2006). Transferencias negativas a nivel morfosintáctico de la lengua materna en el aprendizaje del idioma inglés que se observan en alumnos de educación media. Tesis de Pregrado. Universidad Católica de Temuco.

Denzin, N. (1978). The Research Act. A Theoretical Introduction To Sociological Methods. New York: Mc Graw Hill.

Lasagabaster, D. (1999). (b). Metalinguistic Awareness and The Learning of English as an L3. España: Atlantis XX.

Lasagabaster, D. (2005). La Presencia de Tres Lenguas en el Currículo: Multilingüismo en Los Contextos Canadiense y Español. [Versión electrónica]. Revista de Educación. Vol 337, 405-426.

Lasagabaster, D. Huguet, A. (2007). Multilingualism in European Bilingual Contexts Lanquage Use and Attitudes. Clevedon: Multilingual Matters.

Ludmila, I. (2005). Cross Linguistic Transfer in Word Order: $\quad$ Evidence from L1 Forgetting and L2 Acquisition. Ohio State University: Cascadilla Press.

Ministerio de Educación (1998). Objetivos Fundamentales y Contenidos Mínimos Obligatorios de la Educación Media. Santiago: República de Chile.

Otto, E. (2007). Adquisición de E/LE en un contexto plurilingüe. Transferencias lingüísticas del maltés, inglés e italiano. Consultado el día 2 de Octubre de 2009, en http://elenet.org/Publicaciones/Enciclopedia/Adquisicion\%20de\%20ELE\%20en\%20un\%20c ontexto\%20plurilingueTransferencias

Peláez, A. (2002). Los Mapuche en la Sociedad Chilena Actual. Capítulo 1. Perfil de la Población Mapuche Actual. Santiago: LOM Ediciones. 
Bilingüismo - Trilingüismo - Lingua franca - Mapuche - Mapuzungun - Motivación

Intrínseca - Motivación Extrínseca - Conciencia Metalingüística - Transferencia Lingüística

Pérez, G. (1994). Investigación Cualitativa I. Métodos. Madrid: La Muralla.

Romero, M. (2009). Motivar a aprender en la Universidad: una estrategia fundamental contra el fracaso académico. Aportaciones de la investigación y la literatura especializada. [Versión electrónica]Revista Iberoamericana de Educación. Vol. 50. Consultado en http//www.rieoei.org/deloslectores/3067Ariza.pdf 